H. Sato and Y. Oka

Nagoya Math. J.

Vol. 53 (1974), 189-198

\title{
A CHARACTERIZATION OF UNITARY OPERATORS INDUCED BY NONSINGULAR TRANSFORMATIONS AND ITS APPLICATIONS
}

\author{
HIROSHI SATO AND YUKIMASA OKA
}

In this paper we give a necessary and sufficient condition for a unitary operator on an $L^{2}$-space to be induced by a nonsingular transformation and its applications.

\section{§1. Introduction}

Let $(X, m)$ be a $\sigma$-finite measure space, $L^{2}(m)$ be the complex Hilbert space of all square summable functions and $L^{\infty}(m)$ be the algebra of all bounded measurable functions on $(X, m)$. Then for every $\alpha$ in $L^{\infty}(m)$ we associate a bounded linear operator $T[\alpha]$ on $L^{2}(m)$ by

$$
T[\alpha]: \xi(x) \rightarrow \alpha(x) \xi(x), \quad \xi \in L^{2}(m) .
$$

As is well-known, the correspondence between $L^{\infty}(m)$ and

$$
\Lambda(m)=\left\{T[\alpha] ; \alpha \in L^{\infty}(m)\right\}
$$

is isomorphic and $\Lambda(m)$ is a commutative von Neumann algebra.

A transformation $f$ of $(X, m)$ is a nonsingular transformation if it satisfies the following conditions:

(N.1) There exists a null set $N$ such that $f$ is a bijective transformation of $X-N$ onto itself.

(N.2) $f$ is bimeasurable.

(N.3) $m(E)=0$ if and only if $m \circ f(E)=m(f(E))=0$.

We say $[f ; q]$ is a nonsingular pair if $f$ is a nonsingular transformation and $q=q(x)$ is a complex measurable function such that

$$
|q(x)|^{2}=\frac{d m \circ f}{d m}(x), \quad \text { a.e. }(m) \text {. }
$$

Received October 27, 1973. 
For every nonsingular pair $[f ; q]$ we define a unitary operator $U[f ; q]$ on $L^{2}(m)$ by

$$
U[f ; q]: \xi(x) \rightarrow q(x) \xi(f(x)), \quad \xi \in L^{2}(m) .
$$

In Section 2 we prove the following theorem:

THEOREM 1. Let $(X, m)$ be a $\sigma$-finite abstract Lebesgue space and $U$ be a unitary operator on $L^{2}(m)$. Then there exists a nonsingular pair $[f ; q]$ such that

$$
U=U[f ; q]
$$

if and only if

$$
U^{-1} \Lambda(m) U \subset \Lambda(m)
$$

As applications of Theorem 1, we prove the following theorems in Section 3 and 4 :

THEOREM 3. Let $(X, m)$ be a non-atomic $\sigma$-finite abstract Lebesgue space and $U$ be a unitary operator on $L^{2}(m)$ such that $U-I$ is compact. Then $U$ is induced by a nonsingular pair if and only if $U$ is the identity operator.

This theorem implies that any non-trivial finite dimensional unitary operator on such an $L^{2}$-space is never induced by a nonsingular pair.

THEOREM 5. Let $[f ; q]$ be a nonsingular pair of $\left(\boldsymbol{R}^{1}, d x\right)$ and $\mathfrak{\mho}$ be the Fourier transform. Furthermore, assume that $U[f ; q]$ is a rotation of $\mathscr{S}$, the nuclear space of all rapidly decreasing functions on the real line. Then $\mathfrak{F}^{-1} U[f ; q] \widetilde{\Im}$ is again induced by a nonsingular pair if and only if $[f ; q]$ is given by

$$
\begin{aligned}
& f(x)=\alpha x+\beta, \\
& q(x)=\sqrt{|\alpha|} e^{i(\theta x+\tau)},
\end{aligned}
$$

where $\alpha(\neq 0), \beta, \theta$ and $\tau$ are real constants.

This theorem enables us to construct two one-parameter unitary groups, one of which is induced by nonsingular pairs and the another is not, and still both of them have the same spectral type.

The authors wish to show their hearty thanks to Professor $\mathrm{H}$. Nomoto who gave them valuable suggestions. 


\section{§ 2. Unitary operators induced by nonsingular pairs}

In this section we prove the following theorem:

THEOREM 1. Let $(X, m)$ be a $\sigma$-finite abstract Lebesgue space and $U$ be a unitary operator on $L^{2}(m)$. Then there exists a nonsingular pair $[f ; q]$ such that

$$
U=U[f ; q]
$$

if and only if

$$
U^{-1} \Lambda(m) U \subset \Lambda(m)
$$

Let $(X, m)$ be a $\sigma$-finite abstract Lebesgue space and $[f ; q]$ be a nonsingular pair. Put $q^{\#}(x)=q\left(f^{-1}(x)\right)^{-1}$. Then it is easy to show that $\left[f^{-1} ; q^{\sharp}\right]$ is also a nonsingular pair and

$$
U[f ; q]^{-1}=U\left[f^{-1} ; q^{\sharp}\right] .
$$

Furthermore for every $\alpha$ in $L^{\infty}(m)$ we have

$$
U[f ; q]^{-1} T[\alpha] U[f ; q]=U\left[f^{-1} ; q^{\sharp}\right] T[\alpha] U[f ; q]=T\left[\alpha \circ f^{-1}\right]
$$

and this proves the necessity of Theorem 1.

To prove the sufficiency we make use of the following theorem owed to J. Dixmier.

THEOREM 2. Let $(X, m)$ be a $\sigma$-finite abstract Lebesgue space and $\Phi$ be an automorphism of the commutative von Neumann algebra $\Lambda(m)$. Then there exists a nonsingular transformation $f$ of $(X, m)$ such that

$$
\Phi(T[\alpha])=T\left[\alpha \circ f^{-1}\right]
$$

for every $\alpha$ in $L^{\infty}(m)$.

In J. Dixmier [1], Appendix IV, the above theorem is proved under the different assumption that $X$ is a locally compact topological space with a countable basis and $m$ is a positive Radon measure. On the other hand every abstract Lebesgue space is considered, measure-theoretically, as a pair of such a topological space and a Radon measure (H. Totoki [2]) and we have Theorem 2.

Proof of the sufficiency of Theorem 1. Let $(X, m)$ be a $\sigma$-finite abstract Lebesgue space and $U$ be a unitary operator on $L^{2}(m)$ such that 


$$
U^{-1} \Lambda(m) U \subset \Lambda(m),
$$

that is, for every $\alpha$ in $L^{\infty}(m)$ we have

$$
U^{-1} T[\alpha] U=T[\beta]
$$

for some $\beta$ in $L^{\infty}(m)$. Then it is obvious that

$$
\Phi(T[\alpha])=U^{-1} T[\alpha] U
$$

is an automorphism of $\Lambda(m)$ and by Theorem 2 there exists a nonsingular transformation $f$ of $(X, m)$ such that

$$
\Phi(T[\alpha])=T\left[\alpha \circ f^{-1}\right]
$$

for every $\alpha$ in $L^{\infty}(m)$.

Meanwhile let $V$ be a unitary operator on $L^{2}(m)$ which is induced by a nonsingular pair $\left[f ; \sqrt{\frac{d m \circ f}{d m}}\right]$ of $(X, m)$. Then by a simple estimation we have for every $\alpha$ in $L^{\infty}(m)$

$$
V^{-1} T[\alpha] V=T\left[\alpha \circ f^{-1}\right] .
$$

Consequently we have

$$
V^{-1} T[\alpha] V=U^{-1} T[\alpha] U
$$

and

$$
U V^{-1} T[\alpha]=T[\alpha] U V^{-1}
$$

for every $\alpha$ in $L^{\infty}(m)$. On the other hand, it is well-known that $\Lambda(m)$ is a maximal abelian von Neumann algebra, that is, the von Neumann algebra of all bounded operators on $L^{2}(m)$ that commute with every operator in $\Lambda(m)$. Therefore $U V^{-1}$ belongs to $\Lambda(m)$ and there exists $\gamma$ in $L^{\infty}(m)$ such that

$$
U V^{-1}=T[\gamma] .
$$

Since $U V^{-1}$ is a unitary operator, we have $|\gamma(x)| \equiv 1$ almost everywhere. Define

$$
q(x)=\gamma(x) \sqrt{\frac{d m \circ f}{d m}(x)} .
$$

Then it is easy to verify that $[f ; q]$ is a nonsingular pair of $(X, m)$ and we have 


$$
U=T[\gamma] V=T[\gamma] U\left[f ; \sqrt{\frac{d m \circ f}{d m}}\right]=U[f ; q]
$$

This completes the proof of Theorem 1 .

Note. In Theorem 1 , the criterion is substituted by

$$
U^{-1} \mathfrak{Q} U \subset \Lambda(m),
$$

where $\mathfrak{A}$ is a set of unitary operators on $L^{2}(m)$ which generates $\Lambda(m)$ as a von Neumann algebra.

In particular, if $(X, m)$ is the real line $\left(\boldsymbol{R}^{1}, d x\right)$ or the unit interval $([0,1], d x)$, then it is obvious that $\Lambda(d x)$ is generated from a one-parameter unitary group $\left\{E[t] ; t \in \boldsymbol{R}^{1}\right\}$ defined by

$$
E[t] \xi(x)=e^{i t x} \xi(x), \quad \xi \in L^{2}\left(\boldsymbol{R}^{1}, d x\right),
$$

or from a single operator $E[2 \pi]$ defined by

$$
E[2 \pi] \xi(x)=e^{2 \pi i x} \xi(x), \quad \xi \in L^{2}([0,1], d x),
$$

respectively. Hence we have the following corollaries.

Corollary 1. Let $U$ be a unitary operator on $L^{2}\left(\boldsymbol{R}^{1}, d x\right)$. Then $U$ is induced by a nonsingular pair of $\left(\boldsymbol{R}^{1}, d x\right)$ if and only if

$$
U^{-1} E[t] U \in \Lambda\left(\boldsymbol{R}^{1}, d x\right)
$$

for every $t$ in $\boldsymbol{R}^{1}$.

Corollary 2. Let $U$ be a unitary operator on $L^{2}([0,1], d x)$. Then $U$ is induced by a nonsingular pair of $([0,1], d x)$ if and only if

$$
U^{-1} E[2 \pi] U \in \Lambda([0,1], d x) .
$$

For example, let $\widetilde{\mho}$ be the Fourier transform of $L^{2}\left(\boldsymbol{R}^{1}, d x\right)$ defined by

$$
(\widetilde{\zeta} \xi)(\lambda)=\frac{1}{\sqrt{2 \pi}} \int_{-\infty}^{+\infty} e^{-i \lambda x} \xi(x) d x, \quad \xi \in L^{2}\left(\boldsymbol{R}^{1}, d x\right) .
$$

Then $\mathfrak{F}$ is not induced by any nonsingular pair of $\left(\boldsymbol{R}^{1}, d x\right)$. In fact, assume that $\widetilde{\mho}$ is induced by a nonsingular pair. Then by Corollary 1 there exists a real measurable function $h(x)$ such that

$$
\left(\mathfrak{\mho}^{-1} E[t] \widetilde{\jmath} \xi\right)(x)=e^{i t h(x)} \xi(x), \quad \xi \in L^{2}\left(\boldsymbol{R}^{1}, d x\right)
$$


for every $t$ in $R^{1}$ and almost all $x$ in $\boldsymbol{R}^{1}$. On the other hand, it is wellknown that $\mathfrak{\mho}^{-1} E[t] \widetilde{\mho}$ is a shift transformation. Thus we have

$$
|\xi(x+t)|=|\xi(x)|
$$

for every $t$ in $\boldsymbol{R}^{1}$ and $\xi \in L^{2}\left(\boldsymbol{R}^{1}, d x\right)$, but this is impossible.

\section{§3. In case $U-I$ is a compact operator}

In this section, we prove the following theorem:

THEOREM 3. Let $(X, m)$ be a non-atomic $\sigma$-finite abstract Lebesgue space and $U$ be a unitary operator on $L^{2}(m)$ such that $U-I$ is a compact operator. Then $U$ is induced by a nonsingular pair if and only if $U$ is the identity operator $I$.

Proof. Since the sufficiency is trivial, we prove the necessity. Let $U=U[f ; q]$ be a unitary operator on $L^{2}(m)$ which is induced by a nonsingular pair $[f ; q]$ and $U-I$ be a compact operator. Then, by Theorem 1 , it follows that for every $\alpha$ in $L^{\infty}(m)$, there exists $\beta$ in $L^{\infty}(m)$ such that

$$
U^{*} T[\alpha] U=T[\beta]
$$

Therefore we have

$$
T[\beta-\alpha]=\left(U^{*}-I\right) T[\alpha]+T[\alpha](U-I)+\left(U^{*}-I\right) T[\alpha](U-I)
$$

and consequently $T[\beta-\alpha]$ is a compact operator. Since $m$ is non-atomic, the multiplication operator $T[\beta-\alpha]$ is compact if and only if $\beta-\alpha=0$. This implies that

$$
U^{*} T[\alpha] U=T[\alpha]
$$

for every $\alpha$ in $L^{\infty}(m)$ and consequently $U$ commutes to all multiplication operators $T[\alpha], \alpha \in L^{\infty}(m)$. Since a bounded operator which commutes to all multiplication operators $T[\alpha], \alpha \in L^{\infty}(m)$, is itself a multiplication operator, there exists $\gamma$ in $L^{\infty}(m)$ such that

$$
U=T[\gamma]
$$

By the assumption, the operator $T[\gamma-1]=U-I$ is compact and this implies that $U$ is the identity operator $I$.

This completes the proof. 
We say a unitary operator $U$ on a Hilbert space $\mathscr{H}$ is finite dimensional if there exists a finite dimensional subspace $\mathscr{K}$ of $\mathscr{H}$ such that $U \mathscr{K}=\mathscr{K}$ and $U=I$ on $\mathscr{H} \ominus \mathscr{K}$.

Since it is clear that if $U$ is a finite dimensional unitary operator, then $U-I$ is a compact operator, we have the following corollary of Theorem 3.

COROLlaRy 3. Let $(X, m)$ be a non-atomic $\sigma$-finite abstract Lebesgue space and $U$ be a finite dimensional unitary operator on $L^{2}(m)$. Then $U$ is induced by a nonsingular pair if and only if $U$ is the identity operator $I$.

Let $U$ be a unitary operator on a separable Hilbert space $\mathscr{H}$ and $\left\{\xi_{i}\right\}_{i=1,2, \ldots}$ be an orthonormal basis in $\mathscr{H}$. Representing $U$ as an infinite dimensional unitary matrix $\left(\alpha_{i j}\right)$ by the orthonormal basis $\left\{\xi_{i}\right\}$, where $\alpha_{i j}=\left(U \xi_{i}, \xi_{j}\right), i, j=1,2, \cdots$, it is clear that $U-I$ is a Hilbert-Schmidt operator if and only if

$$
\sum_{i, j=1}^{\infty}\left|\alpha_{i j}-\delta_{i j}\right|^{2}<+\infty
$$

Therefore we have the following corollary.

COROLlaRY 4. Let $(X, m)$ be a non-atomic o-finite abstract Lebesgue space and $U$ be a unitary operator on $L^{2}(m)$ which is represented by a unitary matrix $\left(\alpha_{i j}\right)$ such that

$$
\sum_{i, j=1}^{\infty}\left|\alpha_{i j}-\delta_{i j}\right|^{2}<+\infty
$$

Then $U$ is not induced by a nonsingular pair unless $U$ is the identity operator $I$.

\section{§ 4. Conjugation by Fourier transform}

We say a unitary operator $U$ of $L^{2}\left(\boldsymbol{R}^{1}, d x\right)$ is a rotation of $\mathscr{S}$, the nuclear space of all rapidly decreasing functions on the real line, if the restriction of $U$ to $\mathscr{S}$ is a homeomorphism of $\mathscr{S}$ and denote the group of all rotations of $\mathscr{S}$ by $U(\mathscr{S})$. It is well-known that the Fourier transform $\mathfrak{\mho}$ is a rotation of $\mathscr{S}$.

We say a nonsingular pair $[f ; q]$ of $\left(\boldsymbol{R}^{1}, d x\right)$ is admissible if $U[f ; q]$ is a rotation of $\mathscr{S}$. Then we have the following theorem: 
THEOREM 4. (H. Sato [3], Theorem 2) A nonsingular pair $[f ; q]$ of $\left(\boldsymbol{R}^{1}, d x\right)$ is admissible if and only if it satisfies the following three conditions :

(R.1) $q(x)$ is a slowly increasing function.

(R.2) There exists a positive number $r$ such that

$$
\inf _{x}(1+|x| r)|q(x)|>0 .
$$

(R.3) $f(x)$ is a continuous function and there exists a positive number $\alpha$ such that

$$
\lim _{|x| \rightarrow \infty} \frac{|f(x)|}{|x|^{\alpha}}=+\infty
$$

We say a function on the real line is slowly increasing if it is infinitely differentiable and each of the derivatives is slowly increasing. (R.1) and (R.3) implies that $f(x)$ is a slowly increasing function.

In [3], we consider the following two subgroups of $U(\mathscr{S})$. One is

$$
\text { (B) }=\{U[f ; q] ;[f ; q] \text { is admissible }\}
$$

and another is

$$
\widetilde{S}=\left\{\tilde{U}=\mathfrak{\mho}^{-1} U \mathscr{\mho} ; U \in \mathbb{W}\right\} \text {. }
$$

The intersection of them is not empty (Example 1 4 of [3]) and in the following theorem, we determine the intersection explicitly.

THEOREM 5. Let $[f ; q]$ be an admissible nonsingular pair of $\left(\boldsymbol{R}^{1}, d x\right)$ and $\mathfrak{F}$ be the Fourier transform. Then $\tilde{U}[f ; q]=\mathfrak{\mho}^{-1} U[f ; q] \widetilde{F}$ is again induced by a nonsingular pair if and only if $[f ; q]$ is given by

$$
\begin{aligned}
& f(x)=\alpha x+\beta, \\
& q(x)=\sqrt{|\alpha|} e^{i(\theta x+\tau)},
\end{aligned}
$$

where $\alpha(\neq 0), \beta, \theta$ and $\tau$ are real constants.

Proof. If a nonsingular pair $[f ; q]$ is given as above, then we have

$$
\begin{aligned}
\tilde{U}[f ; q] \xi(x) & =\mathfrak{\mho}^{-1} \sqrt{|\alpha|} e^{i(\theta \lambda+\tau)}(\mathfrak{\mho} \xi)(\alpha \lambda+\beta) \\
& =\frac{\sqrt{|\alpha|} e^{i \tau}}{\sqrt{2 \pi}} \int e^{i \lambda(x+\theta)}(\widetilde{\mho} \xi)(\alpha \lambda+\beta) d \lambda \\
& =\frac{\sqrt{|\alpha|} e^{i \tau}}{\sqrt{2 \pi}} \int \exp \left[i \frac{\lambda-\beta}{\alpha}(x+\theta)\right](\widetilde{\mho} \xi)(\lambda) \frac{d \lambda}{\alpha}
\end{aligned}
$$




$$
\begin{aligned}
& =\frac{\sqrt{|\alpha|}}{\alpha} \exp \left[-i \frac{\beta}{\alpha} x+i\left(\tau-\frac{\beta \theta}{\alpha}\right)\right] \xi\left(\frac{x+\theta}{\alpha}\right) \\
& =U\left[f_{1} ; q_{1}\right] \xi(x)
\end{aligned}
$$

where $\left[f_{1} ; q_{1}\right]$ is a nonsingular pair defined by

$$
\begin{aligned}
& f_{1}(x)=\frac{x+\theta}{\alpha} \\
& q_{1}(x)=\frac{\sqrt{|\alpha|}}{\alpha} \exp \left[-i \frac{\beta}{\alpha} x+i\left(\tau-\frac{\beta \theta}{\alpha}\right)\right]
\end{aligned}
$$

and this proves the sufficiency of the theorem.

To prove the necessity, assume that $\tilde{U}=\tilde{U}[f ; q]$ is induced by a nonsingular pair. Then by Corollary 1 , there exists a real measurable function $H=H(x)$ such that

$$
\tilde{U}^{-1} E[t] \tilde{U} \xi(x)=\widetilde{\mho}^{-1} U\left[f^{-1} ; q^{\sharp}\right] \widetilde{\jmath} E[t] \widetilde{\zeta}^{-1} U[f ; q] \mathfrak{\mho} \xi(x)=e^{i t H(x)} \xi(x)
$$

for every $\xi$ in $\mathscr{S}$. Since $[f ; q]$ is admissible, all these operations are homeomorphisms of $\mathscr{S}$ and by Theorem $4, H(x)$ is infinitely differentiable function with slowly increasing derivatives. Considering $\mathfrak{f} E[t] \mathfrak{\mho}^{-1}$ is the shift $S_{t}: \xi(x) \rightarrow \xi(x-t)$, we have

$$
\frac{q\left(f^{-1}(x)-t\right)}{q\left(f^{-1}(x)\right)} \tilde{\xi}\left(f\left(f^{-1}(x)-t\right)\right)=\widetilde{\mho}\left(e^{i t H} \xi\right)
$$

for every $t$ in $\boldsymbol{R}^{1}$, where $\tilde{\xi}=\widetilde{\jmath} \xi$. Differentiating both sides at $t=0$, we have

$$
\begin{aligned}
& -\frac{q^{\prime}\left(f^{-1}(x)\right)}{q\left(f^{-1}(x)\right)} \tilde{\xi}(x)-f^{\prime}(x) \hat{\xi}^{\prime}(x)=\widetilde{\jmath}(i H \xi) \\
& \quad=\left(T_{H} * \tilde{\xi}\right)(x)=T_{H}(\tilde{\xi}(x-\cdot)), \quad \xi \text { in } \mathscr{S},
\end{aligned}
$$

where $T_{H}$ is the Fourier transform of $\frac{1}{\sqrt{2 \pi}} i H(x)$ in the distribution sense. Since the left side is a value of a distribution supported by a single point $x$ and of order $1, T_{H}$ is a distribution supported by the origin of order 1 and independent of the choice of $x$. Therefore we have

$$
T_{H}=-\alpha \delta^{\prime}-\gamma \delta
$$

where $\delta$ is the Dirac measure and $\alpha$ and $\gamma$ are complex constants. Consequently we have 


$$
f^{\prime}(x) \equiv \alpha, \quad \frac{q^{\prime}\left(f^{-1}(x)\right)}{q\left(f^{-1}(x)\right)} \equiv \gamma
$$

and therefore

$$
f(x)=\alpha x+\beta, \quad q(x)=\rho e^{r x} .
$$

Since $[f ; q]$ is a nonsingular pair, we have

$$
|q(x)|^{2}=|\rho|^{2}\left|e^{r x}\right|^{2}=\left|f^{\prime}(x)\right| \equiv|\alpha| \neq 0,
$$

and $\gamma$ is a pure imaginary number, say,

$$
\gamma=i \theta, \quad \theta \in \boldsymbol{R}^{1}
$$

and

$$
\rho=\sqrt{|\alpha|} e^{i \tau}, \quad \tau \in R^{1} .
$$

Thus we have

$$
f(x)=\alpha x+\beta, \quad q(x)=\sqrt{|\alpha|} e^{i(\theta x+\tau)}
$$

and this completes the proof.

Theorem 5 enables us to construct two one-parameter unitary groups, one of which is induced by nonsingular pairs and the another is not, and still both of them have the same spectral type. For example, oneparameter unitary groups $\left\{U\left[x ; e^{i t x^{3}}\right]\right\}$ and $\left\{\tilde{U}\left[x ; e^{i t x^{3}}\right]\right\}$ are of simple Lebesgue spectrum. But the former is induced by nonsingular pairs and the latter is not.

\section{REFERENCES}

[1] J. Dixmier: Les algèbres d'opérateurs dans l'espace hilbertien. Gauthier-Villars. Paris (1957).

[2] H. Totoki: Introduction to the ergodic theory. Kyoritsu, Tokyo (1971). (in Japanese)

[ 3 ] H. Sato: Rotations of $\mathscr{S}$ induced by nonsingular transformations. (to appear in Memoirs of the Fac. Sci. Kyushu Univ. Ser A, Vol. 28 (1974).

Kyushu University 